\title{
NuRadioMC - Simulation Code for the Next Generation of Radio Neutrino Detectors
}

D. García-Fernández ${ }^{* a, b}$, C. Glaser ${ }^{c}$, A. Nelles ${ }^{a, b}$, J. Alvarez-Muñiz ${ }^{d}$ S. Barwick ${ }^{c}$, D.Z. Besson $^{e}$, S. Buitink ${ }^{f}$, B.A. Clark ${ }^{g}$, A. Connolly ${ }^{g}$, C. Deaconu ${ }^{h}$, K. de Vries ${ }^{f}$, J. Hanson $^{i}$, B. Hokanson-Fasig ${ }^{j}$, S.A. Kleinfelder ${ }^{k}$, R. Lahmann $^{b, c}$, U. Latif ${ }^{e}$, C. Persichilli $^{c}$, Y. Pan ${ }^{l}$, C. Pfender ${ }^{m}$, I. Plaisier ${ }^{a, b}$, D. Seckel ${ }^{l}$, J. Torres Espinosa ${ }^{g}, \mathbf{S}$. Toscano $^{n}$, N. van Eijndhoven ${ }^{f}$, A.G. Vieregg ${ }^{h}$, C. Welling ${ }^{a, b}$, T. Winchen ${ }^{f}$, S.A. Wissel $^{\circ}$

a DESY, Platanenallee 6, 15738, Zeuthen, Germany

b ECAP, Friedrich-Alexander-Universität Erlangen-Nürnberg, Erlangen, Germany

${ }^{c}$ University of California, Irvine, California, USA

${ }^{d}$ IGFAE \& Depto. Física de Partículas, Universidade de Santiago de Compostela, Spain

${ }^{e}$ University of Kansas, Lawrence, Kansas, USA

${ }^{f}$ Vrije Universiteit Brussels, Belgium

${ }^{g}$ CCAPP, Ohio State University, Columbus, Ohio, USA

${ }^{h}$ KICP, University of Chicago, Chicago, Illinois, USA

${ }^{i}$ Whittier College, Whittier, California, USA

${ }^{j}$ University of Wisconsin-Madison, Madison, Wisconsin, USA

${ }^{k}$ Dept. of Electrical Engineering and Computer Science, University of California, Irvine, Irvine, California, USA

${ }^{l}$ University of Delaware, Newark, Delaware, USA

${ }^{m}$ Otterbein University, Westerville, USA

${ }^{n}$ Université Libre de Bruxelles, Brussels, Belgium

${ }^{o}$ Physics Department, California Polytechnic State University, San Luis Obispo, USA

E-mail: daniel.garcia@desy.de

The detection of astrophysical neutrinos by IceCube has opened a new window for the observation of our universe. The use of antennas for detecting astroparticles has been proven useful and accurate after many years of radio cosmic-ray detections, and will be a valuable asset for increasing the statistics for the detection of neutrinos $<10 \mathrm{PeV}$ and reaching out to the highest energies. The radio technique requires realistic and precise simulations of the electric field created after the neutrino interacts with the detector volume, how it propagates through media, and what measurable signal it produces in the detector. With the goal of providing accurate, fast simulations of neutrino-induced electric fields, we have created the NuRadioMC code. It builds on the experience of software used for current radio neutrino and cosmic ray experiments.

36th International Cosmic Ray Conference -ICRC2019-

July 24th - August 1st, 2019

Madison, WI, U.S.A.

${ }^{*}$ Speaker. 


\section{Introduction}

High-energy neutrino astronomy can shed light on the origin of ultra-high-energy cosmic rays (UHECRs). Since we know that UHECRs exist, there must be an associated neutrino flux created upon their interactions with other particles either at their source (astrophysical) or with the CMB during their journey towards us (cosmogenic). Neutrinos are neutral and travel unimpeded due to their low interaction cross section and therefore point directly at their sources.

Precisely due to their low cross section, detecting high-energy neutrinos requires a large interaction volume in a dense medium, such as ice or water. The largest detector that has observed neutrinos, IceCube, has detected neutrinos up to a few PeV energy range [1]. Detection of neutrinos above $10^{16} \mathrm{eV}$ would require a two orders of magnitude increase in sensitivity, which is cost-prohibitive for optical detectors. These neutrinos can be most efficiently observed with the radio technique because of the large attenuation length of radio waves in ice of $>1 \mathrm{~km} \mathrm{[2].}$

When a neutrino interacts in a dense medium a particle shower is produced. As the shower develops in the medium, it acquires a negative charge excess and emits electromagnetic radiation. This is known as the Askaryan effect [4]. This radiation is coherent in the MHz-GHz frequency band, where the Antarctic ice is transparent. A sparse radio array constitutes a cost-effective instrumentation of large effective volumes. Each single station of the array is able to monitor $\sim 1 \mathrm{~km}^{3}$ for ultra-high energy neutrinos.

The radio detection of neutrinos has been successfully piloted with detectors such as ARIANNA [5], ARA [6], and ANITA [7]. After these experimental advances, the radio community is now preparing to build a large radio detector with the size necessary to make a detection likely.

To accompany the experimental improvements, a physically accurate and practical code for calculating the electric field associated to neutrino-induced showers can be a valuable asset. A correct calculation of the electric field allows us to assess the sensitivity of a detector, know its reconstruction capabilities, and optimise it. The Python-based NuRadioMC has been created with that purpose. For a comprehensive documentation of NuRadioMC, see [3].

\section{Structure of NuRadioMC}

NuRadioMC has been created with a modular approach that mimics the actual physics of signal generation, propagation, and conversion to voltage by the antenna electronics. The four pillars of NuRadioMC are:

1. Event generation. The simulation of a neutrino flux and the properties of the subsequent showers. This includes simulation of different flavours, energies, arrival directions. Tau and muon propagation can be taken into account.

2. Signal generation. The calculation of the electric field (Askaryan emission).

3. Signal propagation. The propagation from the emission point to the antenna. This includes a ray-tracing module, needed for media with a depth-dependent refractive index. Attenuation of the electromagnetic waves is also performed. 
4. Detector simulation. The conversion from electric field at the antenna position to the measured final voltage, including trigger simulation. We take into account the frequency-dependent gain of the electronic chain, group delays, sampling rate, etc.

These four pillars, discussed in the following, are complemented by a set of utility classes accessible at all times, such as the modelling of the medium or the signal attenuation.

\section{Event generation}

The event generation module is separated from the rest of the modules, which allows the user to save a specific set of events and study the influence of detector configuration or different physical models for signal emission or ice properties, for instance.

The event generator specifies the neutrino interaction position, the neutrino energy, its flavour, direction, the interaction type (charged current or neutral current), and the inelasticity of the interaction (which determines the cascade energy). All of these parameters are customizable. The neutrino is forced to interact at a given position, and a weight is assigned to take into account the absorption probability after traversing the Earth. All of these parameters are saved in an HDF5 file.

In the case of charged current interactions, muons and taus can be produced. Muons and taus can undergo drastic energy losses along their propagation, which may produce additional showers. Tau decays can also produce showers. These interactions lead to subsequent spatially displaced showers that emit Askaryan radiation, effectively increasing the effective volume of the detector.

Embedded within NuRadioMC is a model of tau decay that includes photonuclear losses, the dominant process at the highest energies, via a continuous slowing down approximation (CSDA). We have also implemented a module that employs PROPOSAL [8], an accurate lepton-propagation code, to obtain the showers induced by tau decays, tau propagation, and muon propagation. We show in Fig.1 the prediction of both approaches for the tau decay energy (left) and length (right). Energy losses along the tau track become relevant around $100 \mathrm{PeV}$. For a $10 \mathrm{EeV}$ tau, the mean energy upon decay is around $0.5 \mathrm{EeV}$, and its mean track length amounts to $50 \mathrm{~km}$. The simple CSDA in NuRadioMC and PROPOSAL agree well on the track length. On the energy, however, there is a noticeable difference due to the drastic energy losses predicted by PROPOSAL and that are not present in the simple model. That translates into a factor of $\sim 5$ for the mean decay energy.

\section{Signal generation}

NuRadioMC allows the user to choose from a variety of frequency-domain parameterisations for the electric field to a time-domain state-of-the-art semi-analytical calculation (see [9]). These are accessible through a unified interface, which also allows for the inclusion of new models.

For effective volume calculations, the Alvarez2009 frequency-domain parameterisation is appropriate due to its fast computation time and the fact that it accurately resembles the predictions of the detailed ZHS Monte Carlo [10,11]. It can be used for hadronic and electromagnetic cascades, as it mimics random fluctuations induced by the LPM effect [11].

The ARZ model [12] uses a semi-analytical approach to calculate the electric field. This module can be used on its own and constitutes a well-tested reference, ported from the original 

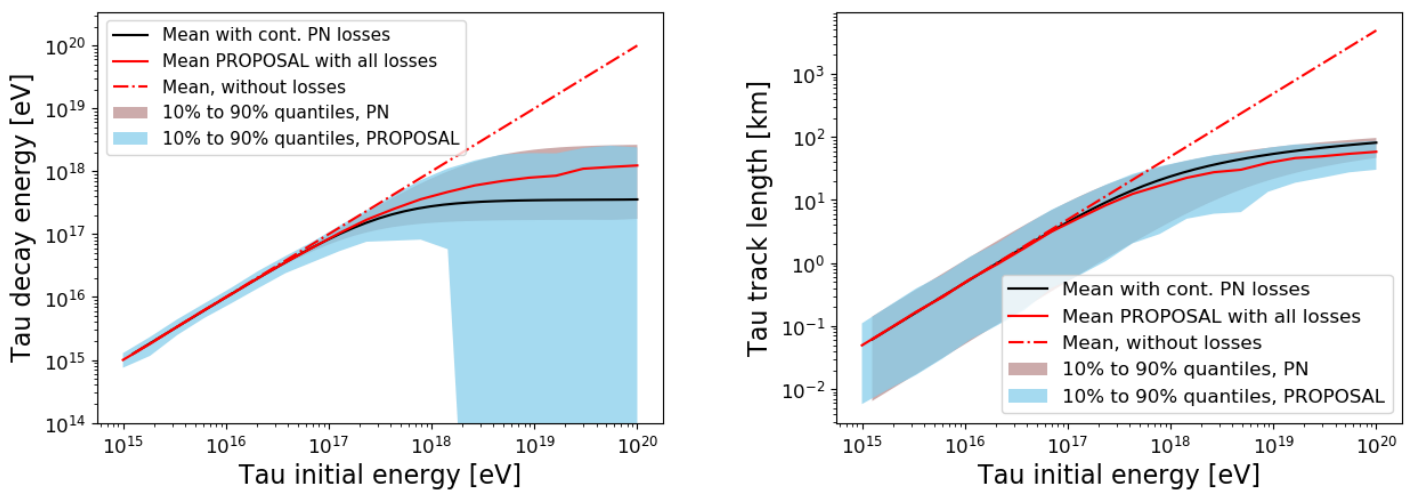

Figure 1: Left: Tau decay energy as a function of the tau initial energy. Right: Tau decay length as a function of the tau initial energy. The shaded bands represent the $10 \%$ to $90 \%$ percentiles for PROPOSAL and the continuous slowing down approximation with photonuclear (PN) losses.

Fortran code. This module calculates the vector potential as an integral containing the shower profile, some geometrical factors, and a form factor common to all showers in dense media, and differentiates to obtain the electric field. The stochastic fluctuations of the shower are included since NuRadioMC is provided with a library of realistic electromagnetic and hadronic shower profiles from the ZHAireS Monte Carlo. This allows a proper treatment of the LPM effect for high-energy electromagnetic showers. This is the most accurate emission model available in NuRadioMC, at the cost of computing time.

We show in Fig. 2 a few charge excess profiles of $10^{17} \mathrm{eV}$ hadronic showers and their associated electric fields according to the ARZ model for two different angles. We show in Fig. 3 some profiles and electric fields for $10^{19} \mathrm{eV}$ electromagnetic showers, which are affected by the LPM effect.

The ARZ model agrees with the ZHS and ZHAireS Monte Carlo at the few percent level up to $2 \mathrm{GHz}$, which is appropriate given the experimental uncertainties. Should more precision be needed, a full Monte Carlo can be implemented in NuRadioMC easily.

\section{Signal propagation}

Signal propagation is treated in NuRadioMC using a propagation module based on an analytic ray-tracing technique that allows for both high speed and accuracy for exponential indexof-refraction profiles, such as $n(z)=n_{\text {ice }}-\Delta_{n} e^{z / z_{0}}$, where $n_{\text {ice }}, \Delta_{n}$ and $z_{0}$ are medium-dependent parameters. The South Pole ice can be described within a few percent using this profile [13].

The analytic ray-tracing module allows for a fast calculation of the travel time of the ray and its bending along the propagation path, which is vital for a correct electric field calculation. The effect of the attenuation along the ray path is calculated numerically using a $\mathrm{C}++$ module [3].

\section{Detector simulation}

The simulation of the detector response to an incoming electric field and the trigger simulation, is performed using NuRadioReco [14], a software for detector simulation and event reconstruction 

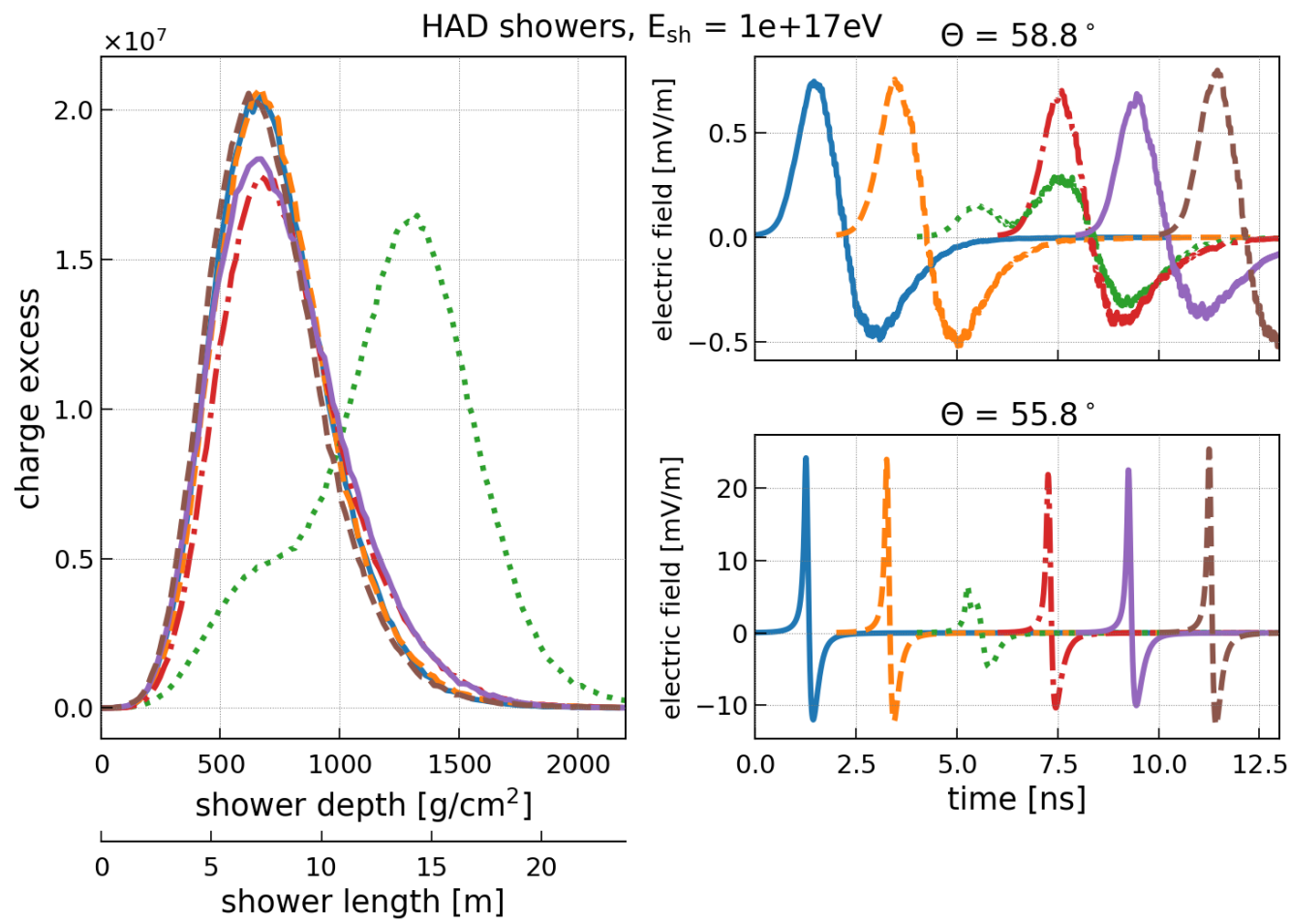

Figure 2: Charge-excess profiles (left) and resulting Askaryan signal according to the ARZ model (right) for several $10^{17} \mathrm{eV}$ hadronic showers in ice and two observation angles w.r.t. shower axis.

of radio neutrino and cosmic-ray detectors.

NuRadioReco provides complete antenna response patterns for commonly used antennas, such as LPDAs, dipoles or bicones, simulated with codes such as WIPL-D and XFdtd. NuRadioReco offers modules to simulate different trigger conditions, as for instance a simple threshold trigger, a high-low trigger (used by ARIANNA) or a phased array trigger (as used by ARA).

\section{Examples}

We proceed to discuss three practical examples of problems we would like to solve for a potential radio array with NuRadioMC to illustrate its capabilities.

\subsection{Calculation of the sensitivity of an Askaryan neutrino detector}

In this example, we calculate the effective volume for a potential radio array. We have chosen a station layout combining 12 LPDAs near the surface having several orientations (horizontal, vertical and tilted) at distances of $\lesssim 10 \mathrm{~m}$ and a borehole with 4 slim dipoles at $15 \mathrm{~m}$ depth in the ice. One million neutrinos per energy bin are generated and simulated in a cylinder of $5 \mathrm{~km}$ radius and $2.7 \mathrm{~km}$ of height, matching the thickness of the South Pole ice layer. The electric field is calculated using the Alvarez2009 model and the detector simulation is performed (see tutorial in [15]).

Once the simulation is complete, the triggering information contained in the output files can be used to retrieve the sensitivity. The sensitivity of the detector is quantified in terms of effective 

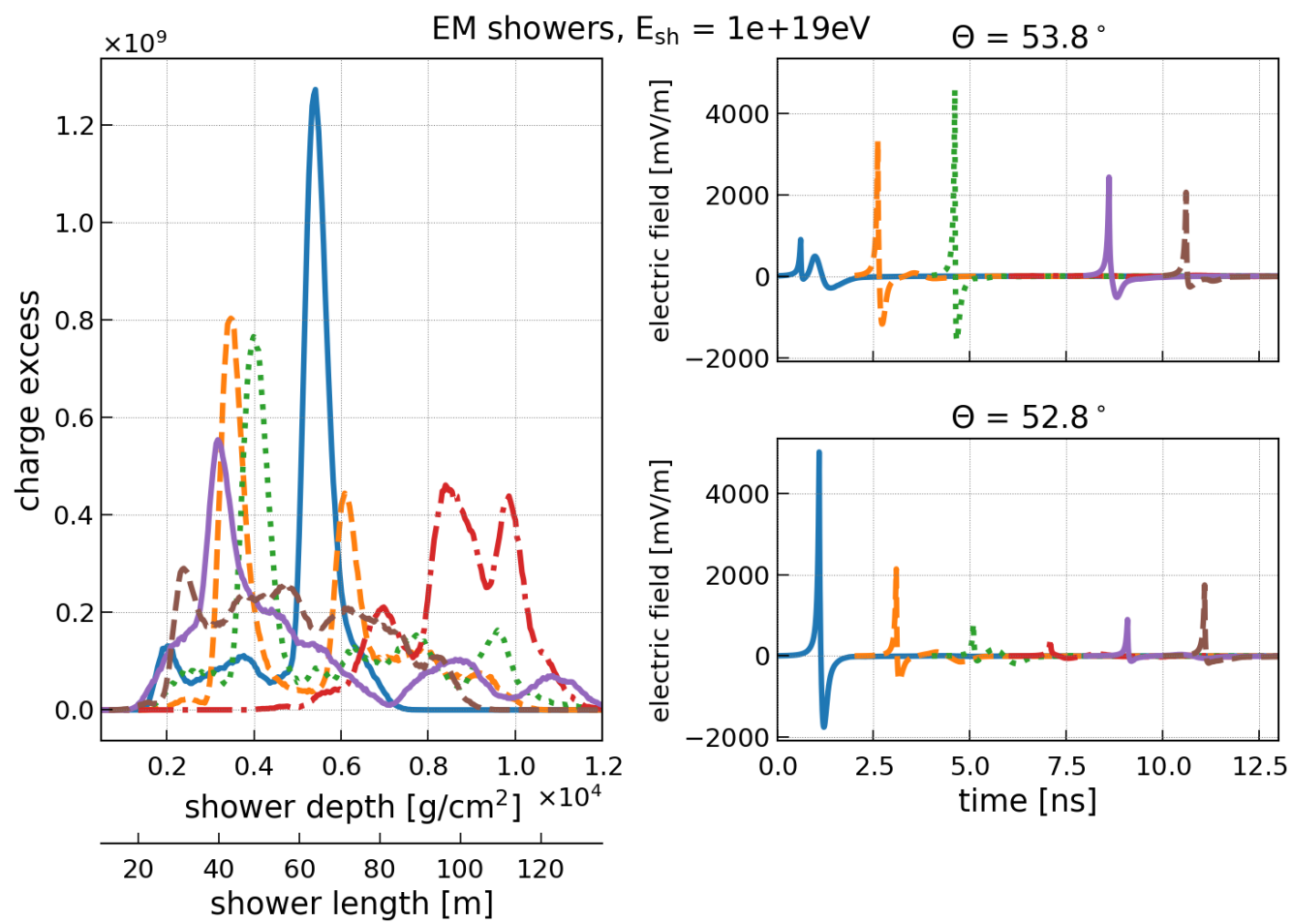

Figure 3: Charge-excess profiles (left) and resulting Askaryan signal according to the ARZ model (right) for several $10^{19} \mathrm{eV}$ electromagnetic showers in ice and two observation angles w.r.t. shower axis. The presence of several peaks in the profile is due to the LPM effect

volume to an isotropic neutrino flux. We calculate a weighted sum of all triggering events, divide by the total number of events, and multiply by the geometrical volume times the solid angle of the incoming events ( $4 \pi$ in our case). The weights used are the probability of neutrino survival after having traversed the Earth and reached the simulation volume. The results for the effective volume are shown in Fig. 4 (left). This effective volume is then transformed into effective area with the interaction length and converted into a limit on the diffuse neutrino flux for our detector, using the 90\% CL Feldman-Cousins formula for no events and no background, also shown in Fig. 4 (right).

\subsection{Calculation of the efficiency to detect both the direct and reflected electric fields}

In this example we calculate the efficiency of the detection of both the direct and reflected pulses. Electric fields from particle showers in ice are reflected on the ice surface. Consequently, the observation of the direct and the reflected electric field for a single neutrino event is possible, which is a signature that offers major reconstruction advantages, since the vertex position is better constrained with two pulses instead of one. The ray-tracing module in NuRadioMC possesses a complete description of reflection on the ice surface. We show the efficiency to detect both pulses for $10^{18} \mathrm{eV}$ energy neutrinos in Fig. 5, left. The efficiency is largest for shallow antennas and decreases with depth. This is because of the finite width of the Cherenkov cone. The deeper the antenna is placed, the smaller the chance that both signal paths have a similar signal amplitude. However, deep antennas provide more effective volume for direct events. 

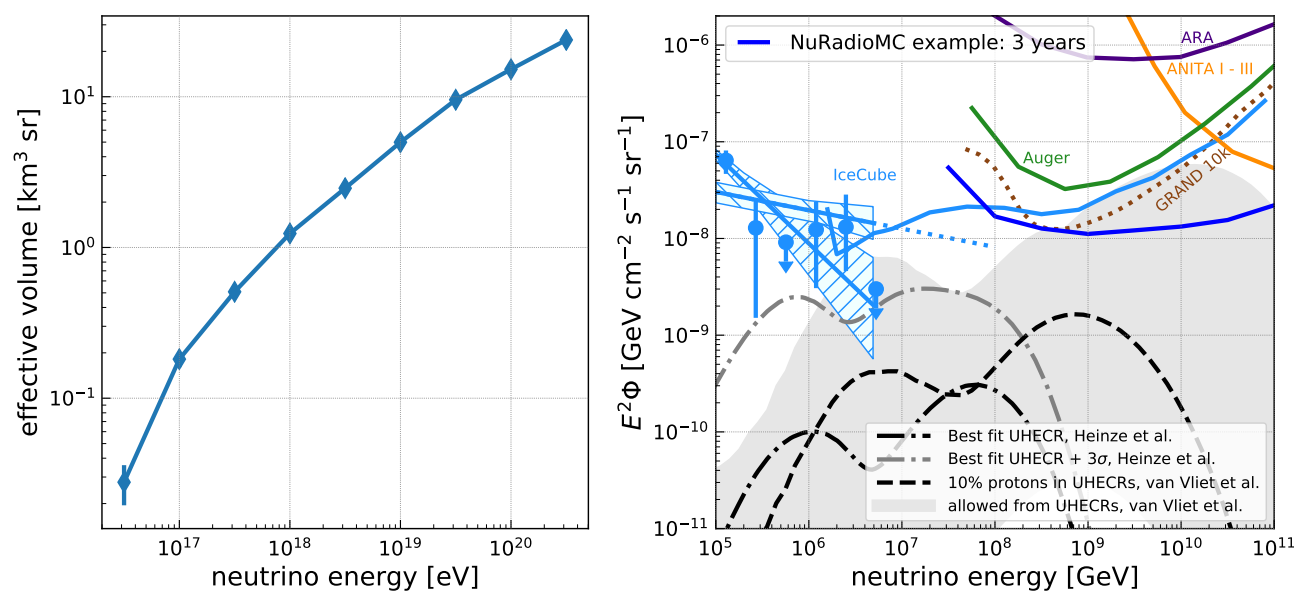

Figure 4: Left: effective volume of a sample detector station as a function of energy. Right: Corresponding expected limit for a diffuse neutrino flux for a detector comprising 100 stations and an uptime of 3 years, compared to current experimental limits.
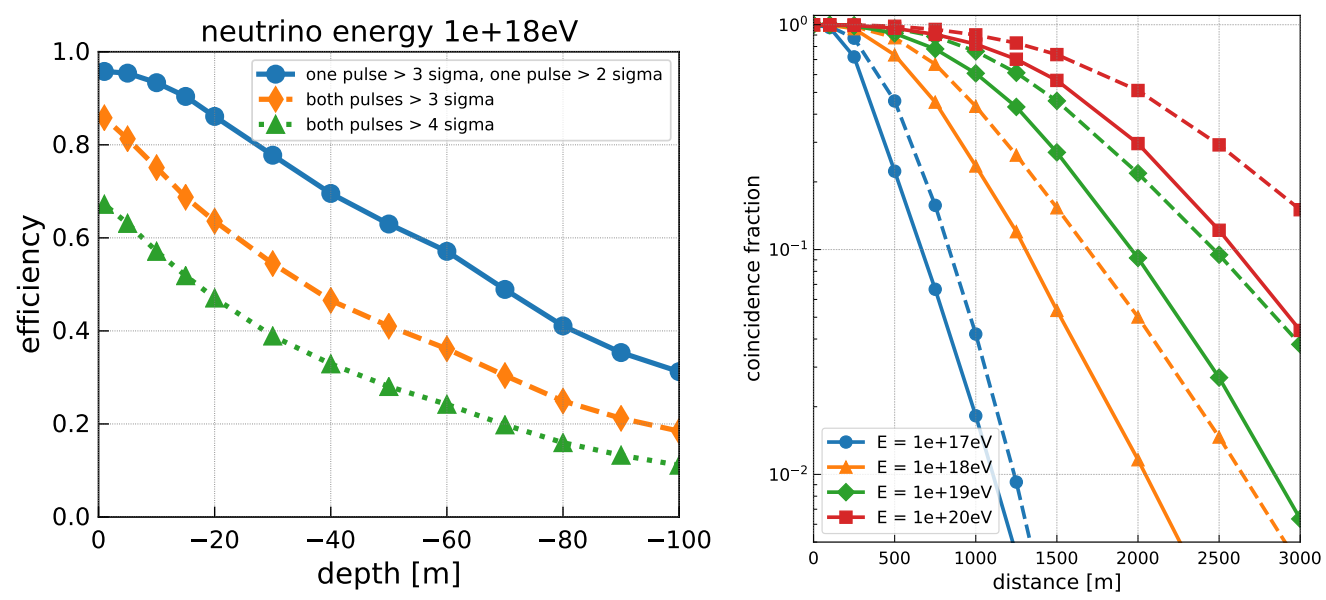

Figure 5: Left: Efficiency of the detection of both the direct and reflected Askaryan pulses as a function of antenna depth for a neutrino energy of $10^{18} \mathrm{eV}$ and different thresholds. Right: Probability of detecting the same neutrino event in multiple stations as a function of the distance between stations. Solid line is for a surface detector, dashed line for a $50 \mathrm{~m}$ deep detector.

\subsection{Optimisation of station spacing for an Askaryan neutrino detector}

We can also use NuRadioMC to calculate the probability to detect a signal from the same neutrino in multiple stations of an array. We have chosen an LPDA surface array and a $50 \mathrm{~m}$ deep dipole to undertake this study. We create an eight-arm star-shaped array [3] with these two types of stations, placing them at various distances (from $100 \mathrm{~m}$ to $3 \mathrm{~km}$ ) from the centre. We simulate the electric field for neutrinos of several energies and obtain their triggering events. After these, the triggers for each station (signal three times above noise RMS) are compared and the events that triggered more than one station are identified. The results are shown in Fig. 5, right. To maximise the overall sensitivity of a detector, coincidences between stations should be minimised which requires separation distances of more than $1.5 \mathrm{~km}$. On the other hand, to improve reconstruction one 
may want several coincidences. Both optimisation problems can be studied using NuRadioMC. The actual effective volume per station depends on the antenna positions, but it can be approximated, counting only nearest-neighbour and 2-station coincidences, as:

$$
V_{\mathrm{eff}}=V_{\mathrm{eff}, 0}\left(1-\frac{p_{2} N_{2}}{N_{\mathrm{s}}}\right) \approx V_{\mathrm{eff}, 0}\left(1-2 p_{2}\left(1-\frac{1}{\sqrt{N_{s}}}\right)\right)
$$

where $V_{\text {eff, } 0}$ is the effective volume of an isolated station, $p_{2}$ is the probability of a 2-station coincidence, $N_{\mathrm{s}}$ is the number of stations, and $N_{2}$ is the number of nearest-neighbour pairs in the array. We have supposed a square array, so that $N_{2}=2\left(N_{\mathrm{s}}-\sqrt{N_{\mathrm{s}}}\right)$.

\section{Conclusions}

We have discussed NuRadioMC [3], a framework for simulating radio neutrino detectors. It provides state-of-the-art models for event generation, signal generation, signal propagation and detector simulation. We have briefly discussed these modules and some examples of the capabilities NuRadioMC offers. NuRadioMC combines the experience acquired after previous cosmic-ray and neutrino experiments. Its versatility and its embedded tools can help proposed radio experiments such as RNO, ARIA, GRAND, ANITA/PUEO or BEACON [16, 17, 18]

\section{References}

[1] IceCube collaboration, Astrophys. J. 809 (2015) 98

[2] S. Barwick et al., J. Glaciol. 51 (2005) 173

[3] C. Glaser et al., arXiv:1906.01670. Submitted to EPJ-C

[4] G.A. Askaryan, JETP 21 (1965) 658

[5] The ARIANNA collaboration, IEEE Trans. Nucl. Sci. 62 (2015) 2202

[6] The ARA collaboration, Phys. Rev. D 83 (2016) 083003

[7] P.W. Gorham et al., Astropart. Phys. 32 (2009) 10

[8] J.-H. Koehne et al., Comput. Phys. Commun. 1849 (2013) 2070

[9] NuRadioMC, https://github.com/nu-radio/NuRadioMC/wiki/Interface-to-Askaryan-modules

[10] J. Alvarez-Muñiz, C.W. James, R.J. Protheroe, E.Zas, Astropart. Phys. 326 (2009) 100

[11] J. Alvarez-Muñiz, W.R. Carvalho Jr., M. Tueros, E.Zas, Astropart. Phys. 356 (2012) 287

[12] J. Alvarez-Muñiz, A. Romero-Wolf, E.Zas, Phys. Rev. D 81 (2010) 123009

[13] S.W. Barwick et al., JCAP 7 (2018) 55

[14] C. Glaser et al., Eur. Phys. J C 79 (2019) 464

[15] NuRadioMC tutorial, https://github.com/nu-radio/NuRadioMC/wiki/Tutorial

[16] GRAND collaboration, Science and Design 1810.09994

[17] A. Karle and A. Nelles, in Proceedings of the XVIII International Workshop on Neutrino Telescopes, Venice, 2019

[18] S.A. Wissel, in Proceedings of ARENA 2018, Catania, 2018 